\title{
Article
}

\section{Effects of ecstasy/polydrug use on memory for associative information}

\author{
Gallagher, Dennis T., Fisk, John, Montgomery, Catharine, Judge, \\ Jeannie, Robinson, Sarita Jane and Taylor, Paul John \\ Available at http://clok.uclan.ac.uk/4229/ \\ Gallagher, Dennis T., Fisk, John ORCID: 0000-0002-2981-0870, Montgomery, \\ Catharine, Judge, Jeannie ORCID: 0000-0002-4029-6876, Robinson, Sarita Jane \\ ORCID: 0000-0002-4237-5412 and Taylor, Paul John ORCID: 0000-0002-9999- \\ 8397 (2012) Effects of ecstasy/polydrug use on memory for associative \\ information. Psychopharmacology, 222 (4). pp. 579-591. ISSN 0033-3158
}

It is advisable to refer to the publisher's version if you intend to cite from the work. http://dx.doi.org/10.1007/s00213-012-2652-x

For more information about UCLan's research in this area go to http://www.uclan.ac.uk/researchgroups/ and search for < name of research Group>.

For information about Research generally at UCLan please go to http://www.uclan.ac.uk/research/

All outputs in CLoK are protected by Intellectual Property Rights law, including Copyright law. Copyright, IPR and Moral Rights for the works on this site are retained by the individual authors and/or other copyright owners. Terms and conditions for use of this material are defined in the policies page.

\section{CLoK}

Central Lancashire online Knowledge www.clok.uclan.ac.uk

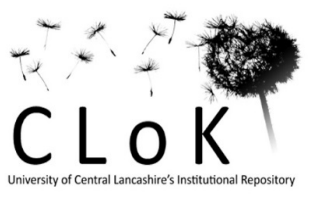


Effects of Ecstasy/Polydrug Use on Memory for Associative Information

Running Head: Ecstasy and Associative Information

Denis T. Gallagher ${ }^{1}$, John E. Fisk ${ }^{1}$, Catharine Montgomery ${ }^{2}$, Jeannie Judge ${ }^{1}$, Sarita J. Robinson ${ }^{1}$, Paul J. Taylor ${ }^{1}$

\author{
${ }^{1}$ University of Central Lancashire, Preston PR1 2HE, United Kingdom \\ ${ }^{2}$ Liverpool John Moores University, Liverpool, L3 3AF, United Kingdom
}

Corresponding author:

Professor John E. Fisk, PhD

School of Psychology,

Darwin Building,

University of Central Lancashire,

Preston, Lancs. PR1 2HE

United Kingdom

Tel 44 (0) 1772894465

Fax 44 (0) 1772892925

Email: JFisk@uclan.ac.uk

\title{
Disclosures and Acknowledgements
}

The authors declare that, except for income received from their primary employers, this research received no specific grant from any funding agency in the public, commercial, or not-for-profit sectors. All authors contributed significantly to this report and have read and approved the final manuscript. The authors are not aware of any conflict of interest and do not have any financial interest in this piece of research. 


\section{Abstract}

Rationale. Associative learning underpins behaviours that are fundamental to the everyday functioning of the individual. Evidence pointing to learning deficits in recreational drug users merits further examination. Objectives. A word pair learning task was administered to examine associative learning processes in ecstasy/polydrug users. Methods. After assignment to either single or divided attention conditions, 44 ecstasy/polydrug users, and 48 nonusers were presented with 80 word pairs at encoding. Following this, four types of stimuli were presented at the recognition phase; the words as originally paired (old pairs), previously presented words in different pairings (conjunction pairs), old words paired with new words, and pairs of new words (not presented previously). The task was to identify which of the stimuli were intact old pairs. Results. Ecstasy/ploydrug users produced significantly more false positive responses overall compared to nonusers. Increased long-term frequency of ecstasy use was positively associated with the propensity to produce false positive responses. It was also associated with a more liberal signal detection theory (SDT) decision criterion value. Measures of long term and recent cannabis use were also associated with these same word pair learning outcome measures. Conjunction word pairs, irrespective of drug use, generated the highest level of false positive responses and significantly more false positive responses were made in the DA condition compared to the SA condition. Conclusions. Overall, the results suggest that long-term ecstasy exposure may induce a deficit in associative learning and this may be in part a consequence of users adopting a more liberal decision criterion value.

Key Words: Ecstasy, Drug Use, Cognition, Memory, Associative Learning, Word Pairs 
Despite the acute positive behavioural and emotional changes that follow ecstasy (MDMA) use, users also demonstrate significant impairment on a range of cognitive tasks including measures of verbal (Bolla et al. 1998; Klugman et al. 1999; McCardle et al. 2004) and non-verbal learning and recall (Klugman et al. 1999), executive functioning (Fisk et al. 2004; Montgomery et al. 2005b) and working memory (Wareing et al. 2004; Morgan et al. 2002; Verdejo-García et al. 2005). As both executive functioning and working memory underlie associative learning it was suggested that learning processes in MDMA users may also been impaired. Evidence for an ecstasy/polydrug-related deficit in associative learning was observed by Montgomery et al. (2005a) and the present paper seeks to further explore this deficit by examining recognition and associative learning in MDMA users.

Associative learning refers to a conscious attentional process whereby associations are formed between two previously unrelated stimulus representations (Montgomery et al. 2005a). Failure to adequately bind stimuli to form new associations within the context of their occurrence can have obvious implications for the individual. By way of illustration, in the context of learning to drive, we are required to form appropriate associations between several visual stimuli and specific behaviours, for example, we need to learn to pair a red traffic light signal with a stop action. With each association there is a need to form a connection between two previously unrelated stimuli until the required behaviour becomes automatic. However, the successful learning and acquisition of such skills is highly dependent on the working memory system and executive functions in that there is a requirement for the effective use of strategies and self-monitoring meta processes (Montgomery et al. 2005a; Tanji and Hoshi 2001). Given that ecstasy use is accompanied by working memory deficits (Montgomery et al. 2005b; Morgan et al. 2002; Verdejo-García et al. 2005; Wareing et al. 2004), we may expect that ecstasy users will be less able to bind features of different stimuli together to form new associations.

Aside from the role of the working memory system in associative learning, research suggests that that there may be a significant dependency on a broad range of pre-frontal cortical and medial-temporal hippocampal structures (Passingham et al. 2000; Moscovitch 2000; Moscovitch and Winocur, 1992). For example, Passingham et al. (2000) had participants learn associations between visual stimuli and specific motor actions. Their results suggest that the PFC plays a crucial role in integrating information about the stimulus, the required response and the associated motor action. Indeed it appears that representations of 
the stimuli and the response are both mapped onto areas of PFC, and that the formation of associations between the two is mediated by interconnections within this region (Passingham et al. 2000). In a further examination of regional brain involvement in associative learning, Law et al. (2005) investigated patterns of brain activity in humans who completed a visuospatial associative learning task (pairing an abstract image with a spatial location). They observed increases in activity during learning in several frontoparietal regions whilst the association was being encoded. However, once the association had been learnt, activity in these areas returned to baseline. In contrast, activity in the medial temporal lobe (including in the hippocampus and parahippocampal cortices) increased monotonically during learning. Thus it appears that both PFC and medial-temporal structures are fundamental to the acquisition of new associations with the former playing a key role in the initial learning of associations and the latter in their consolidation. Interestingly, research suggests that both these areas may be susceptible to the effects of illicit drugs. For example, there is evidence to suggest that ecstasy use is associated with deficits in tasks which recruit prefrontal executive resources (see the review by Murphy, et al. 2009). Cannabis on the other hand has been shown to potentially compromise the functioning of the medial temporal region (e.g., Jager et al. 2007; Lawston et al. 2000; Messinis et al. 2006).

In view of research which has demonstrated ecstasy-related degradation in regions of the PFC (Sekine et al. 2003) and hippocampus (Thompson et al. 2004), and research showing ecstasy-related impairment within the working memory system (Fisk et al. 2004; Montgomery et al. 2005b; Wareing et al. 2004), it is not surprising that ecstasy users have poorer memory for associative information relative to nonusers (Croft et al. 2001b; Gouzoulis-Mayfrank et al. 2003; Montgomery et al. 2005a). Using a paired associated learning (PAL) task, Croft et al. (2001b) asked a sample of ecstasy and cannabis users, cannabis only users and drug naive counterparts to learn associations between six spatially defined stimulus pairs (“spatial”) or six colour pairings (“non-spatial”). While no significant differences were found between the ecstasy/cannabis users and the cannabis only users, when both drug using samples were combined, the resulting group performed significantly worse than nonusers on the non-spatial PAL task. However, subsequent ANCOVA revealed that this effect was more attributable to cannabis than ecstasy. While apparently implicating cannabis rather than ecstasy it is worthy of note that the ecstasy users in Croft et al.'s study had a relatively low lifetime exposure to the drug and as such were atypical of users participating in other studies (for example, see Morgan et al. 2002). 
In another study that also used a spatial PAL task, Fox et al. (2002) presented participants with six boxes situated in a circle on a computer screen. As each box opened, an abstract pattern was revealed. In a recognition phase, participants were presented with a single abstract pattern in the centre of the screen and asked to identify in which of the six boxes it had first appeared. The task consisted of two trials, where participants were required to learn six (Trial 1) and eight (Trial 2) pattern-location pairs. While there were no group differences on the majority of outcome measures, for number of errors, the group by trial interaction approached significance with post- hoc tests confirming that ecstasy users made more errors than nonusers on the eight pair trial.

Roberts et al. (2009) administered a face-number associative learning task in which participants learned to associate specific numbers with particular faces over a number of trials. Relative to cannabis-only controls and drug free persons, ecstasy users performed significantly worse overall averaged over all trials. During the performance of the task, fMRI revealed that ecstasy users displayed higher levels of activation in a number of brain regions including the left superior frontal gyrus, [BA (Brodman areas) 6 and 9], the middle frontal gyrus bilaterally, (BA 10 and 11) as well as bilateral temporal and occipital lobe structures. In addition, right hemisphere parietal lobe structures also exhibited higher levels of activation in ecstasy users. In contrast, ecstasy users had a number of regions exhibiting lower levels of activation (including the right anterior cingulate and the left posterior cingulate and parahippocampal gyrus). Both cannabis and ecstasy users exhibited lower levels of activation in the right medial frontal gyrus and left parahippocampalgyrus. It is possible that elevated activity levels in the prefrontal cortex are caused by this area taking over functions normally served by more posterior structures.

Studies specifically examining word-pair associative learning in ecstasy/polydrug users have produced ambiguous results with apparent ecstasy-related effects confounded with other factors (Gouzoulis-Mayfrank et al. 2003) or due to other drugs (Montgomery et al. 2005a) or only apparent under more demanding conditions (Brown et al. 2010). In those studies where deficits have been observed ecstasy users' failure to form paired associates as readily as nonusers might be attributable to a number of factors. For example, they may simply be unable to recall the response word. Alternatively, there might be a tendency for users to mismatch cues and responses by producing the associate of a different cue word. In this sense ecstasy users might be unable to maintain the original pair binding. This is in line with Kroll et al.'s (1996) theory that false memories are formed when we inappropriately recombine previously presented information to form episodes that have not occurred. 
Ultimately, this leads to the incorrect binding of processed information and causes memory conjunction errors. Such errors are often revealed when people are subjected to several stimuli types including pictures, faces and word pairs (Reinitz et al. 1996).

These possible explanations for the observed deficit in associative learning in ecstasy users have not yet been systematically explored. Therefore the current study aims to further explore the underling ecstasy-related impairments which give rise to the previously observed deficits in associative learning. We will also seek to gain further evidence regarding the potential contributions of cannabis and other illicit drugs in accounting for the associative learning deficits that have been observed.

With this aim in mind it is proposed to make use of a word pair learning task (see Castel and Craik 2003) that provides several measures of error thereby providing a better understanding of ecstasy-related deficits in associative learning. For example, having previously studied the original pairings during the encoding phase, participants will be presented with four types of stimuli during the recognition phase. These will include old pairings (both words presented together as originally paired in the encoding phase), conjunction word pairs (both words presented in the encoding phase but in different pairings), item word pairs (previously presented words paired with new ones), as well as entirely new word pairs (both words not previously presented), thereby providing a contextual element to the task. In relation to the first two of these, although both word pair types contain two previously presented stimuli, in one case, words are presented within the same pair context (old), and in the other case, words are presented in an entirely new pair context (conjunction). Kroll et al.'s (1996) proposal of false memory formation might suggest that ecstasy users are more susceptible to binding failures and as such, form high numbers of false memories in situations where previously presented items of information are recombined to form episodes that have not occurred (conjunction word pairs). The subsequent “memory conjunction errors" may be a result of the incorrect combination of processed information in ecstasy users. Another possible explanation is that nonusers are better than ecstasy users in utilising contextual information to identify previously presented stimuli within the context of their occurrence. By exploring differences in the recall of old and conjunction word pairs, we will be able to investigate the extent to which ecstasy users and nonusers differentially use context information to enhance recognition performance. Specifically, we expected ecstasy users to be less efficient than nonusers in binding stimuli (i.e., words) together within the context of their occurrence (i.e., their pairing). If this were the case, ecstasy users should demonstrate inferior recognition of old word pairs compared to nonusers. We may also expect ecstasy 
users to make more false positive responses towards conjunction and possibly item word pairs than nonusers.

The use of a recognition based paradigm like that developed by Castel and Craik (2003) also allows the use of signal detection theory to analyse the proportions of hits and false positive responses. Thus the degree of sensitivity exhibited in detecting previously seen stimuli that are differentially activated can be directly examined. Similarly the willingness to produce an affirmative response to ambiguous stimuli can also be assessed through examination of the decision criterion value. Lower sensitivity will result in fewer hits while a lower decision criterion value will give rise to more false positive responses. To the authors' knowledge this technique has not been previously used in studies of the effects of ecstasy/polydrug use on memory and learning.

In addition to investigating the processes underpinning associative learning under the usual single task conditions, the word pair learning task used in this study also includes a dual-task condition, a digit monitoring task during encoding, which is expected to place an additional load on executive processes (see Logie et al. 2004). Previous research has indicated that the division of attention impairs performance on associative learning tasks (Reinitz et al. 1994). If deficits in ecstasy users result primarily from executive functioning impairment in general (rather than associative impairment in particular) then the difference between the hits to old pairs in the divided attention and single attention conditions should be greater in ecstasy users compared to controls. Similarly, in relation to the conjunction parings, as a consequence of the additional executive load, both groups will produce more false positive responses in the divided attention condition compared to the single attention condition but this deficit will be more evident among the ecstasy user group. This is because the PFC is involved in binding the original pairs during encoding and so in the divided attention condition it should be more difficult to effectively bind the pairs together resulting in more misses in relation to the old pairings and more false alarms for the conjunctive and possibly the item pairings. It is possible that a similar pattern will emerge although to a lesser degree for the old items paired with new ones. However, the recognition of new items should be unaffected by the dual task at encoding since the items were never originally presented.

Thus to summarise, it is predicted that all participants will produce more false positive responses in the conjunction pairs compared to the item and entirely new pairings. This tendency is expected to be especially pronounced among ecstasy users and more so under conditions of divided attention. Thus a three way interaction is predicted between drug users group (ecstasy/polydrug versus non ecstasy user), type of pairing presented at recognition 
(conjunction, item and new) and dual task (present or absent). In relation to the proportion of hits and false positive responses, a signal detection analysis will be conducted. It is predicted that ecstasy users will exhibit less sensitivity and a more liberal decision criterion. In addition to the between group comparison, various measures of long term illicit drug use will be correlated with the number of positive responses, hits for the old pairs, and false positive responses for the conjunction, item, and new pairs. In particular it is expected that measures of ecstasy use will be negatively correlated with the number of hits, positively correlated with false positive responses and negatively correlated with sensitivity and decision criterion.

\section{Method}

Design

In order to investigate whether ecstasy/polydrug users generate fewer correct responses to old pairs, the number of hits for old-old pairs (the dependent variable) will be analysed in a two way design with drug user group (ecstasy/polydrug versus non ecstasy user), and dual task (present or absent) both between participants. The same independent variables will be used for the signal detection outcomes to investigate whether ecstasy/polydrug users differ from nonusers on the sensitivity and decision criterion measures but with both these dependent variables included together in a multivariate analysis of variance. For the main analysis, to examine whether ecstasy/polydrug users relative to nonusers have a greater tendency to produce false positive responses specifically to conjunction pairs and whether this tendency is exacerbated in the dual task condition, a three way design will be used with drug user group (ecstasy/polydrug versus non ecstasy user), and dual task (present or absent) between participants and type of pairing presented at recognition (conjunction, item and new) within participants. The dependent variable was the number of affirmative 'yes', i.e., false positive, responses. In order to explore the difference between conjunction pairs and the average of the other word pair types (item and new) orthogonal Helmert contrasts will be used. The same contrasts will be used within the relevant interactions to establish whether this difference varies significantly between user groups and attention condition. The advantage of orthogonal contrasts is they that partition the available degrees of freedom thereby allowing differences between cell means to be explored without inflating the Type 1 error rate (Tabachnick and Fidell, 2007). To supplement this various measures of illicit drug use will be correlated with hits, the three classes of false positive responses (conjunction, item and new) and with the sensitivity and decision criterion measures. 


\section{Participants}

Ninety-two undergraduate students (44 ecstasy users, mean age $=22.50$ and 48 nonusers, mean age=20.96) from the University of Central Lancashire and Liverpool John Moores University participated in the study. Students were recruited via an online participant recruitment scheme (Sona-Systems) and by the snowball technique (Solowij et al. 1992). All students received a $£ 20$ grocery store gift card for their participation in the study. Students of Liverpool John Moores University who were recruited via the online participant recruitment scheme also received course credits for their participation.

Participants were asked to refrain from ecstasy use in the 10 days prior to the test session (the median period of abstinence was in fact 12 weeks). Participants were also asked to refrain from the use of other illicit substances, including cannabis, ketamine and cocaine for at least 24h before the test-session. They were randomly assigned to participate in one of two conditions [Single Attention (SA); 23 ecstasy users and 21 nonusers, and Divided Attention (DA); 22 ecstasy users and 27 nonusers].

\section{Materials}

The use of ecstasy and other drugs was assessed by means of a self-report questionnaire previously used in several studies from our laboratory (e.g., Fisk et al. 2009). For each year since they commenced drug use, participants estimated the typical dose that they ingested in a representative session. They also estimated their typical frequency of use (number of sessions per week) during that year. This was done for all illicit drugs that were regularly consumed during each specific year. This allowed estimates of long term dose (averaged over the number of years of use) and similarly the long term average frequency of use to be computed. In addition a measure of variability for each of these measures was computed, specifically, in each case, the standard deviation of the annual data. These annual estimates were also used to produce an estimate of total lifetime use. Participants also indicated their current frequency of use and the period of abstinence for each major illicit drug. Demographic variables including age and gender were recorded and fluid intelligence was measured through Raven’s progressive matrices (Raven et al. 1998).

The word pair learning task was closely based on one developed by Castel and Craik (2003). In the encoding phase, a total of 160 common concrete nouns were paired to form 80 word-pairs. Each participant was visually presented with all 80 word-pairs in a randomized order in the centre of 15.4-in computer screen. Word-pairs were presented for a total of 4s 
with a 500ms gap between presentations. The encoding phase was immediately followed by a recognition phase where participants were presented with a further 60 word-pairs in a randomized order. Word-pairs consisted of previously presented, old word pairs and newly presented, conjunction, item and new word-pairs.

Old word-pairs contained two words that were previously seen as a pair in the encoding phase. Conjunction word-pairs contained two words that were previously presented in the encoding phase, but not as an original pair. Item word-pairs contained one word that was presented in the encoding phase in the first position and a new word in the second position. New word-pairs contained a new word in both the first and second positions. Each of the four word-pair types appeared a total of 15 times during the recognition phase. Once again, word pairs were presented for a total of $4 \mathrm{~s}$ with a 500-ms gap between presentations.

A digit-monitoring task was introduced during the encoding phase of the DA condition. The digit- monitoring task was programmed using E-Prime and involved the simultaneous presentation of a series of digits ranging from 0 to 9 . A total of 240 auditory digits were presented in a randomized order. The participant's task was to make a predetermined response (i.e., pressing the "Y" key on a computer keyboard) after the presentation of 3 three consecutive odd digits (e.g., 1, 3, 5, or 5, 7, 3).

\section{Procedure}

All participants provided informed verbal consent. The research was approved by the Ethics Committees of both institutions and was conducted in accordance with the ethical guidelines of the British Psychological Society. Ecstasy users and nonusers were randomly assigned to either a SA or DA condition. At the beginning of both conditions, participants were visually presented with a set of instructions on a computer screen. Participants were informed that they would be presented with a number of word-pairs and asked to remember each word and it's pairing for a recognition test. Further instructions were provided for participants in the DA condition. Participants were informed that they would receive an auditory presentation of a series of digits ranging from 0 to 9 . Participants were asked to listen to the digits and make a response using the "Y" key on a computer keyboard after the presentation of 3 consecutive odd digits.

After the completion of the encoding phase, participants were visually presented with a set of instructions for the recognition phase. The recognition phase consisted of 60 wordpairs as described in the materials section above. Using a computer keyboard to make responses, participants were instructed to identify previously seen (old) word pairs by 
pressing the "Y" key and to respond to newly presented (conjunction, item, and new) wordpairs by pressing the " $\mathrm{N}$ ” key.

\section{Results}

As is evident from the data in Table 1, intelligence (Raven's progressive matrices), years of education, and alcohol and cigarette use did not differ significantly between the two groups. However, age did differ significantly between ecstasy users and nonusers. With regard to illicit drugs, ecstasy users differed from nonusers on the long term average dose and frequency of use of cannabis as well as total cannabis use with ecstasy users recording significantly higher values on all of these variables.

$$
<<\text { Insert Table } 1 \text { about here }>>
$$

\section{Word Pair Recognition Task}

In the word pair recognition test, “old” responses to previously presented word pairs are classified as hits, while "old” responses to any type of newly presented word pair (conjunction, item and new) were considered false alarms. Table 2 shows the mean number of "old" responses made to each of the four word pair types (old, conjunction, item, new) by ecstasy users and nonusers in the SA and DA conditions.

\section{$<<$ Insert Table 2 about here $>>$}

Inspection of Table 2 suggests that non users tended to correctly recognise more "old" words pairs than ecstasy users in the single attention condition. However, by way of contrast ecstasy users recognised more previously presented word pairs in the DA condition. Despite this, compared to nonusers, ecstasy users were more likely to report that they had seen all word pair types in the DA condition. In general, ecstasy users made more false alarms than nonusers to newly presented word pairs (conjunction, item, new), however, this trend was more apparent in the DA condition.

To investigate the effects of ecstasy use and attention condition (SA or DA) on the number of “old” responses to previously presented word pairs, a 2-way ANOVA was performed on the relevant data shown in Table 2. ANOVA did not reveal a significant effect of ecstasy use $(F(1,89)=.16, p=.70)$ or attention condition $(F(1,89)=2.58, p=.12)$ on number of hits recorded. Further, the interaction between ecstasy use and attention condition on number of hits was not significant, $F(1,89)=2.58, p=.11$.

To investigate effects of word pair type, ecstasy use and attention condition on false alarm errors, a 3 (conjunction, item, new) x 2 (ecstasy user/nonuser) x 2 (SA or DA) mixed ANOVA was performed on the relevant data shown in Table 2. Significant main effects were 
found for ecstasy use $\left(F(1,89)=4.32, p<.05\right.$, partial $\left.\eta^{2}=.05\right)$ with users producing significantly more false positives overall compared to nonusers. There was also a main effect of word pair type $\left(F(2,89)=16.91, p<.001\right.$, partial $\left.\eta^{2}=.16\right)$, with the conjunction pairs generating the highest level of false positives, item pairs rather fewer and new pairs the smallest number of false positives. Helmert test of within-subject contrasts (word pair type) revealed that significantly more false alarms were made towards conjunction word pairs relative to item and new-word pairs together $\left(F(1,89)=27.03, p<.001\right.$, partial $\left.\eta^{2}=.23\right)$. In addition, attention condition yielded a significant main effect $(F(1,89)=22.93, p<.001$, partial $\eta^{2}=.21$ ) with more false positive responses being made in the DA condition.

With regard to the predicted three way interaction the trends in the relevant means are set out in Figure 1. Despite the divergent trends evident in the left and right panels the predicted crucial three way interaction was non-significant, $\mathrm{F}<1$, and there were no other significant interactions between the variables, $\mathrm{F}(2,178)=2.14$, $\mathrm{p}=.12$, for word pair type by attention condition, and $\mathrm{F}(1,89)=1.70, \mathrm{p}=.20$, for user group by attention condition, and $F(2,178)=1.92, p=.15$, for word pair type by user group. However, while the overall interaction between user group and word pair type was non-significant, it is worthy of note that one of the interaction contrasts between ecstasy use and word pair type approached significance. This contrast compares the difference between false positive responses to conjunction pairs and (averaged) false positive responses to the other two word pair types for ecstasy users with the same difference for nonusers. This comparison revealed that ecstasy users' tendency to produce more false positive responses than nonusers was more evident in the conjunction condition relative to the group difference in other two conditions, $F(1,89)=3.11, p=.081$. Consistent with this, post hoc analyses (with an adjusted alpha $=.016$ ) revealed that ecstasy users produced significantly more conjunction false positive responses compared to nonusers, $\mathrm{p}=.014$, while the two groups did not differ significantly in the number of item, $\mathrm{p}=.684$, and new false positive responses, $\mathrm{p}=.228$.

\section{$<<$ Insert Figure 1 about here $>>$}

The signal detection variables, sensitivity (d prime) and decision criterion, were included as dependent variables in a multivariate analysis of variance (MANOVA) with drug user group and attention condition as independent variables. The effect of user group was not significant, $\Lambda=.950, \mathrm{~F}(2,88)=2.34, \mathrm{p}>.05$ and neither was the user group by attention condition interaction $\Lambda=.973, \mathrm{~F}(2,88)=1.23$, $\mathrm{p}>.05$. However, there was a significant effect of attention condition, $\Lambda=.795, \mathrm{~F}(2,88)=11.36, \mathrm{p}<.001$. The univariate outcomes revealed that the effect of attention condition was statistically significant for the sensitivity measure, 
$F(1,89)=16.43, p<.001$. The effect approached significance for the decision criterion $F(1,89)=3.56, p=.062$. Both sensitivity and decision criteria were lower in the divided attention condition. While the multivariate effect of user group failed to reach significance, the univariate outcome for the decision criterion approached significance, $F(1,89)=3.35$, $\mathrm{p}=.070$. Ecstasy/polydrug users had a lower decision criteria compared to nonusers.

\section{Correlation data}

As is apparent from inspection of Table 3, the long-term average frequency of ecstasy use was positively and significantly correlated with false positive responses towards both conjunction and item word pairs, $p<.01$, and $p<.05$, respectively. In addition the same measure was negatively correlated with the signal detection decision criterion value, $p<.01$; and contrary to prediction was positively correlated with the number of hits in relation to old word pairs, $p<.01$. Other statistically significant outcomes included the variability in the long-term average frequency of ecstasy use which was positively correlated with the number of false positive responses towards conjunction and new word pairs, and negatively correlated with the signal detection decision criterion value, $p<.01$ in all cases. As was the case with the basic measure, contrary to prediction, the variability in the long-term average frequency of ecstasy use was positively correlated with the number of hits for old word pairs, $p<.01$. None of the other ecstasy use measures, i.e., long term average dose (level and variability), current frequency of use, and total lifetime consumption, were significantly associated with the number of hits or false positive responses. The unexpected statistically significant positive association between the number of hits and the average level and variability of the long term frequency of ecstasy use might be mediated by the negative association between these two variables and the decision criterion value. In order to evaluate this possibility, the partial correlations between the number of hits and, respectively, the average level and variability of the long term frequency of ecstasy use, were computed controlling for the decision criterion measure. The results revealed that neither partial correlation was statistically significant, $\mathrm{r}_{\mathrm{p}}=.110$ and -.015, for hits and, respectively, the average level and variability of the long term frequency of ecstasy use, p>.05 in both cases.

$<<$ Insert Table 3 about here $>>$

With regard to the other drugs, the variability in the long-term average dose of cannabis use was positively and significantly correlated with false positive responses towards conjunction, $p<.01$, and item word pairs, $p<.05$ and negatively associated with the signal detection decision criterion value, $p<.05$. Current frequency of cannabis use was also 
positively correlated with false positive responses towards conjunction, $p<.01$ and new word pairs, $p<.05$. The long-term average frequency of cocaine use and the variability in this measure were positively and significantly correlated with false positive responses for item word pairs, $p<.05$ in both cases. None of the other drug use measures for cannabis and cocaine were significantly associated with the number of hits or false positive responses.

In order to establish whether or not the indicators of illicit drug use were associated with unique variance in the word pair responses, partial correlations were conducted controlling for the variance shared between the word pair outcomes and any other illicit drugs that possessed statistically significant zero order correlations. The results are set out in Table 4. Thus, for example, in the first column of Table 4, the partial correlation, .431, between the long term variability in the average dose of cannabis and the number of conjunction false positive responses, is following controls for the long term average frequency and variability in the frequency of ecstasy use. Similarly, in the same column, the partial correlation, .263, between the long term average frequency of ecstasy use and the number of conjunction false positive responses, is following controls for variability in the long term average cannabis dose and the current frequency of cannabis use. Inspection of Table 4 reveals that measures of both cannabis and ecstasy use share unique variance with conjunction false positive responses and with the SDT decision criterion value. Variability in the long term average frequency of ecstasy use also shares unique variance with false positive responses to new word pairs. While a number of the other partial correlations were not statistically significant, this does not necessarily imply that the particular drug in question has no effect on word pair learning, rather that the pooled variance shared with other illicit drugs prevent a judgement as to which particular drug is responsible for the significant zero order correlations that were observed.

$$
<<\text { Insert Table } 4 \text { about here }>>
$$

\section{Discussion}

The findings of the present study are in concert with those of other studies of associative learning in drug user populations (Brown et al. 2010; Montgomery et al. 2005a; Roberts et al. 2009) and support the view that ecstasy use may induce deficits in memory for associative information. In general, ecstasy users performed worse than nonusers in recognising and rejecting new word pairings and this was reflected in higher rates of false alarms to conjunction and new word pairs in the SA condition and to conjunction, item and new word pairs in the DA condition. 
As expected from previous research, both groups made more false positive responses towards conjunction word pairs compared to item and new word pairs together and both groups made significantly more errors in the DA condition compared to the SA condition. More importantly in accordance with our prediction, overall, ecstasy users made significantly more false positive responses compared to nonusers. The interaction contrast between word pair type and group approached significance reflecting the fact that while all participants were more likely to produce false positive responses for conjunction pairs relative to item and new, this tendency was especially pronounced among ecstasy users. This is consistent with expectation since the binding of old word pairs recruits executive resources that are known to be impaired among ecstasy users (Montgomery et al., 2005b; Verdejo-García et al., 2005; Wareing et al., 2004). Given that dual task paradigms are suggested to load on executive resources (Logie et al. 2004), it was expected that the pattern underpinning this 2-way interaction would be especially pronounced in the DA condition. However, this was not the case. While ecstasy users produced more false positive responses for conjunction pairs in the dual task condition they also exhibited the same tendency for the item and new pairs. Thus ecstasy users' ability to detect the recombined conjunction pairs was similarly compromised in both the single and dual task conditions. One possible explanation is that dual task performance loads on qualitatively different executive resources than those employed in the binding of old word pairs. This proposal is in line with Miyake et al.’s (2000) assertion that dual task performance does not load on to any of their original conceptualizations of executive functions (shifting, updating, and inhibition). Nonetheless, it is possible that the simultaneous completion of two tasks is mediated by some other aspect of executive function which has yet to be delineated. Examination of Figure 1 reveals that the group difference in false positive responses was greater in the dual task condition suggesting that ecstasy users are impaired in terms of the executive processes which support dual task performance. However since the group by task interaction was non-significant further research is needed to explore this possibility.

Contrary to prediction, overall, ecstasy users and nonusers did not differ in the number of hits that were achieved. This may be because ecstasy users adopted a more liberal decision criterion and so were more likely at recognition to endorse ambiguous stimuli as previously intact old pairs. This tendency might allow users to offset the effects of any underlying impairment in associative learning but at the cost of producing more false positive responses. While plausible this interpretation must be treated with a degree of caution since the group difference on the decision criterion variable was just short of significance on a twotailed basis. 


\section{Correlation data}

The absence of a relationship between total prior consumption of ecstasy and word pair learning performance confirms previous findings from our laboratory (Montgomery et al. 2005a) and is consistent with the proposition that total number of tablets consumed is not always directly related to neurotoxic dose (O’Shea et al. 1998). Estimates of lifetime use may not capture subtle differences in consumption patterns. Rather it may be other aspects of long term use such as typical dose and frequency of use that are important. Thus while small doses over a long period of use and large doses for a relatively short period may produce similar lifetime exposure the cognitive consequences might be radically different. That is not to say that total use is wholly unimportant as a potential indicator of neurotoxicity. The possibility of a long term cumulative effect of the drug cannot be ruled out. For example, in an ERP study, Croft et al. (2001a) found that lifetime dose of ecstasy was associated with 5-HT dysfunction after partially out the effects of variations in the current frequency of use while there was no association between the frequency of use and 5-HT when the effects of differences in lifetime does were controlled. Similarly other studies examining different aspects of cognition have found a significant lifetime dose related effect in relation to ecstasy (e.g., Indlekofer et al. 2009; Schilt et al. 2008). Nonetheless, in the present study, it appears that ecstasy related impairment in associative learning is associated with more refined and explicit measures of ecstasy use. For example, higher average long-term frequencies and the long-term variability in the frequency of ecstasy use were associated with superior recognition of previously presented word pairs. Whilst this is not in line with our initial predictions, both of these long-term measures of ecstasy use were negatively correlated with the signal detection variable, decision criterion. As such, it might be that ecstasy users made a greater number of "Yes" responses to old word pairs (hits) but also to conjunction, item and new word pairs (false alarms). Thus rather than reflecting superior performance, it is likely that the positive correlation between the long term indices of ecstasy use and number of hits is a consequence of an increased likelihood of positive responses across trials. The results of the partial correlations controlling for the decision criterion value which reduced the association between the ecstasy use variables and the number of hits to below statistical significance supports this proposition. As expected, long term measures of ecstasy use were positively correlated with false positive responses towards conjunction, and new word pairs.

Aside from ecstasy related impairments in associative learning, the current study highlights several aspects of other drug use that may affect memory for associative 
information. In particular, increased variability in the long term average dose of cannabis and higher current frequencies of cannabis use were associated with increased levels of false positive responses to newly presented word pairs. Furthermore, higher long term average frequencies of cocaine use and higher variability in long-term average frequency of cocaine use were associated with increased false positive responses specifically to item word pairs. The correlation data summarised above highlights several dimensions of illicit drug use that may affect associative learning performance and may provide an explanation of the inconsistency between the existing findings in the literature.

Prior to the present study, there had been few investigations specifically into word pair learning among ecstasy/polydrug users. Gouzoulis-Mayfrank et al. (2003) used the wordpair learning subset of the LGT-3 (Baumler 1974), where subjects were required to memorise 20 word pairs containing a Turkish word in the first position its German translation in the second position. In an immediate and delayed recognition phase, participants were presented with a German word and were required to identify its Turkish translation from five possible options. Heavy ecstasy users were significantly worse than nonusers in identifying correct word pairs in the delayed recall condition, but not in the immediate recall condition. However, this effect did not remain significant following the inclusion of general knowledge scores as a covariate.

In order to better understand the basis of associative learning deficits in ecstasy users, Montgomery et al. (2005a) used a verbal paired-associates learning task that included measures of forgetting, perseverative errors and speed of associative leaning (trials to completion). Participants were visually presented with eight sequential word pairs after which they were presented with the first word from each pair and asked to recall the second word from its original pairing. Eight such learning trials were administered. Relative to nonusers, ecstasy users demonstrated significantly poorer performance on each of the measures noted above. Nevertheless, attributing these findings solely to the use of ecstasy is problematic. In this study, for example, cannabis use in particular was shown to be an important determinant of initial learning and forgetting.

Brown et al. (2010) administered a variety of cognitive tasks including word pair learning and word triplet learning. In the former, participants studied pairs of unrelated words after which they were presented with the first member of each pair and required to recall the associate. Although there were ceiling effects in later trials, in the initial learning trial ecstasy users were significantly impaired relative to a cannabis-only control group and the impairment in relation to nondrug users approached significance. In word triplet learning 
participants studied three words, after which the first word was presented with the participant required to recall the remaining two words. The task was repeated five times and ecstasy users were consistently impaired relative to nondrug users over all five trials. Interestingly, cannabis users performed similarly to ecstasy users in trial one but by trial five their performance had improved to the same level as the drug naïve group.

Thus to summarise there is a degree of ambiguity in previous research into word pair learning among ecstasy users. Brown et al.’s (2010) ecstasy users performed worse than nonusers but the difference only approached significance. Similarly, Gouzoulis-Mayfrank et al.'s (2003) results failed to demonstrate a clear ecstasy-related effect. It is also the case that in the present study ecstasy users did not differ in terms of the number of hits for old word pairs, but they did generate significantly more false positive responses for the other pairs perhaps as a consequence of adopting a less stringent decision criterion. Thus participants in the Brown et al. (2010) and the Gouzoulis-Mayfrank et al. (2003) studies may have maintained their performance by using a similar strategy. In Montgomery et al.’s (2005a) study it was unclear that the deficits observed in word pair learning were attributable to ecstasy use. In the present study, the use of more refined indicators of long term drug use provides stronger grounds for attributing at least some of the effects that were observed to aspects of ecstasy use. Nonetheless it is necessary to exercise caution in attributing observed effects to specific drugs. Since in many cases the same individuals consumed both cannabis and ecstasy and since the use of these two drugs appears to co-vary, it is not possible to be definitive in assigning the effects observed here to a specific substance. Furthermore, while the partial correlations reported in Table 4 demonstrate that the long term frequency of ecstasy use appears to share unique variance with false positive responses and the SDT decision criterion variable, it is also worthy of note that the variability in the long term average dose of cannabis also shared unique variance with these variables.

A number of limitations need to be acknowledged in relation to the present study. As in much of the existing literature this study has relied on self-report data in relation to drug use. Clearly it would have been desirable to confirm abstinence through the urinalysis or some alternative means. However, studies which have compared self-reported drug use to other independent measures, such as biomarkers, have found a respectable level of reliability and validity (Darke 1998) and so the use of self-report does not necessarily undermine the results of the current study. 
With regard to the statistical analysis, it must be conceded that the correlations reported in Table 3 are unadjusted for multiple comparisons. With full Bonferroni correction none of the correlations reported in Table 3 are significant at the adjusted alpha level. However, each drug use indicator is correlated with multiple outcome measures (i.e., the different types of false positive response and the SDT variables) which are significantly inter-correlated each other. The situation is further constrained by the fact that there are multiple drug use indicators which are themselves inter-correlated. In such a situation, full Bonferroni correction is too conservative and inappropriate (Sankoh et al. 1997) and there is no universally accepted method for calculating the adjusted alpha level. Nonetheless some degree of correction is appropriate and in these situations and an adjusted alpha level of .01 is sometimes adopted (e.g., Montgomery and Fisk 2007). At this level, seven of the correlations would be statistically significant. Another indicator would be the error rate per experiment (Howell 1997) which utilising the Bonferroni criteria, would produce between 5 and 6 randomly significant outcomes at an unadjusted alpha level of .05 $(108 \times .05)$. In fact ignoring the outcomes which were contrary to prediction, 13 of the correlations were significant.

A further method for evaluating the importance of the outcomes reported in Table 3 would be to utilise Cohen's (1988) effect size construct. According to this measure, correlations of less than 0.1 are unlikely to represent a noteworthy effect, correlations of between 0.1 and up to 0.3 represent a small effect size, from 0.3 up to 0.5 moderate, and correlations 0.5 and above a large effect size. Using this standard, those correlations in Table 3 which are significant at $\mathrm{p}<.01$, one tailed, all exceed 0.3 and so would meet Cohen's criteria for a moderate effect. From an alternative perspective Tabachnick and Fidell (2007) recommend a cut of .32 (corresponding to $10 \%$ shared variance) as being indicative of a potentially meaningful relationship between a factor and a variable loading on it.

Nonetheless, on these criteria, while a number of correlations in Table 3 might be worthy of note, it is important to acknowledge that with an adjusted alpha level of .01 as a cut-off point, the proportion of variance shared between the drug use indicators set out in Table 3 and the source memory outcomes is modest ranging from $10 \%$ to $16 \%$ and for the SDT item memory outcomes from $19 \%$ to $21 \%$. Thus the practical implications for illicit drug users in terms of source memory performance are perhaps modest in scale and should not be overstated.

In conclusion, the findings from the present study coincide with previous research and confirm assumptions that long-term ecstasy exposure may induce a deficit in memory for associative information. However, more refined and explicit measures of ecstasy use have 
been identified that appear to be more clearly associated with these deficits. In particular, higher long-term average frequency and the long-term variability in the frequency of ecstasy use were both associated with increased false positive responses towards newly presented word pairs. In the broader context of associative learning, the present results suggest that there may be a tendency for ecstasy users to select the wrong response for any given cue thereby mismatching stimulus response pairings during the early phases of learning. Thus responses may be equally well learned but paired with the wrong stimulus. It is possible that nonusers may be better able to use contextual information to form associations between specific items.

It is worthy of note that the present results relate to verbal learning and we acknowledge that binding may be differentially affected by the type of stimuli presented during encoding and as such, drug users may be more susceptible to binding failures according to the nature of the stimuli encoded at presentation. Future studies may wish to utilise alternative forms of visual stimuli, for example, male and female faces or abstract patterns to address the true extent of associative learning deficits in drug users. 
References

Baumler G (1974) Lern-und Gedachtnistest (Learning and memory test) LGT-3 Verlag fur Psychologie Hogrefe, Gottingen

Bolla KI , McCann UD, Ricaurte GA (1998) Memory impairment in abstinent MDMA (“Ecstasy”) users. Neurology 51: 1532-1537

Brown J, McKone E, Ward J (2010) Deficits of long-term memory in ecstasy users are related to cognitive complexity of the task. Psychopharmacology 209: 51-67

Castel AD, Craik FIM (2003) The effects of aging and divided attention on memory for associative information. Psychol Aging 18: 873-885

Cohen, J. (1988). Statistical Power Analysis for the Behavioral Sciences (2nd ed.). New Jersey: Lawrence Erlbaum.

Croft RJ, Klugman A, Baldeweg T, Gruzelier JH (2001a) Electrophysiological evidence of serotonergic impairment in long-term MDMA (“ecstasy”) users. Am J Psychiatry 158: $1687-1692$

Croft RJ, Mackay AJ, Mills ATD, Gruzelier JGH (2001b) The relative contributions of ecstasy and cannabis to cognitive impairment. Psychopharmacology 153: 373-379

Darke S (1998) Self-report among injecting drug users: A review. Drug Alcohol Depen 51: 253-263

Fisk JE, Montgomery C, Murphy PN (2009) The association between the negative effects attributed to ecstasy use and measures of cognition and mood among users Exp Clin Psychopharm 17: 326-336

Fisk JE, Montgomery C, Murphy C, Wareing M (2004) Evidence for executive deficits among users of MDMA (Ecstasy). Brit J Psychol 95: 457-466

Fox HC, McLean A, Turner JJD, Parrott AC, Rogers R, Sahakian BJ (2002) Neuropsychological evidence of a relatively selective profile of temporal dysfunction in drug-free MDMA (“ecstasy”) polydrug users. Psychopharmacology 162: $203-214$

Gouzoulis-Mayfrank E, Thimm B, Rezk M, Hensen G, Daumann J (2003) Memory impairment suggests hippocampal dysfunction in abstinent ecstasy users. Prog Neuropsychoph 27: 819-827

Howell DC (1997) Statistical Methods for Psychology ( $4^{\text {th }}$ ed). Belmont CA: Duxbury 
Indlekofer F, Piechatzek M, Daamen M, Glasmacher C, Lieb R, Pfister H, Tucha O, Lange KW, Wittchen HU, Schütz CG (2009) Reduced memory and attention performance in a population-based sample of young adults with a moderate lifetime use of cannabis, ecstasy and alcohol. J Psychopharmacol 23: 495-509

Jager G, Van Hell HH, De Win MML, Kahn RS, Van den Brink W, Van Ree, JM, Ramsey NF (2007). Effects of frequent cannabis use on hippocampal activity during an associative memory task. Eur Neuropsychopharmacol 17: 289-297

Klugman A, Hardy S, Baldeweg T, Gruzelier J (1999) Toxic effect of MDMA on brain serotonin neurons. Lancet 353: 1269

Kroll NEA, Knight RT, Metcalfe J, Wolf ES, Tulving E (1996) Cohesion failure as a source of memory illusions. J Mem Lang 35: 176-196

Law JR, Flanery MA, Wirth S, Yanike M, Smith AC, Frank LM, Suzuki WA, Brown EN, Stark CEL (2005) Functional Magnetic Resonance Imaging Activity during the Gradual Acquisition and Expression of Paired-Associate Memory. J Neurosci 25: 5720-5729

Lawston J., Borella A., Robinson JK, \& Whitaker-Azmitia PM (2000). Changes in hippocampal morphology following chronic treatment with the syntheticcannabinoid WIN 55,212-2._Brain Res 877: 407-410.

Logie RH, Cocchini G, Della Sala S, Baddeley AD (2004) Is there a specific executive capacity for dual task coordination? Evidence from Alzheimer’s disease. Neuropsychology 18: 504-513

McCardle K, Luebbers S, Carter JD, Croft R J, Stough C (2004) Chronic MDMA (ecstasy) use, cognition and mood. Psychopharmacology 173: 434-439

Messinis L, Kyprianidou A, Malefaki S, Papathanasopoulos P (2006) Neuropsychological deficits in long-term frequent cannabis users. Neurology 66: 737-739.

Miyake A, Friedman NP, Emerson MJ, Witzki AH, Howerter A (2000) The unity and diversity of executive functions and their contributions to complex "frontal lobe" tasks: A latent variable analysis. Cognitive Psychol 41: 49-100

Montgomery C, Fisk JE (2007) Everyday memory deficits in ecstasy-polydrug users. J Psychopharmacol 21: 709-717

Montgomery C, Fisk J E, Newcombe R (2005a) The nature of ecstasy-group related deficits in associative learning. Psychopharmacology 180: 141-149 
Montgomery C, Fisk J E, Newcombe R, Murphy P N (2005b) The differential effects of ecstasy-polydrug use on executive functions: Shifting, inhibition, updating and access to semantic memory. Psychopharmacology 182: 262-276

Morgan MJ, McFie L, Fleetwood LH, Robinson JA (2002) Ecstasy (MDMA): are the psychological problems associated with its use reversed by prolonged abstinence? Psychopharmacology 159: 294-303

Moscovitch M (2000) Theories of memory and consciousness In E Tulving \& F I M Craik (Eds), The Oxford Handbook of Memory (pp 609-625). New York: Oxford University Press

Moscovitch M, \&Winocur G (1992) The neuropsychology of memory and aging. In F IM Craik \& T A Salthouse (Eds), The Handbook of Aging and Cognition (pp 315-372) Hillsdale, NJ: Erlbaum

Murphy PN, Wareing M, Fisk JE, \& Montgomery C (2009). Executive Working Memory Deficits in Ecstasy/MDMA Users: A Critical Review. Neuropsychobiology 60:159175.

O’Shea E, Granados R, Esteban B, Colado MI, Green AR (1998) The relationship between the degree of neurodegeneration of rat brain 5-HT nerve terminals and the dose and frequency of administration of MDMA ('ecstasy'). Neuropharmacology 37: 919-926

Passingham RE, Toni I, Rushworth MFS (2000) Specialisation within the prefrontal cortex: the ventral prefrontal cortex and associative learning. Exp Brain Res 133: 101-113

Raven J, Raven JC, Court JH. (1998) Manual for Raven’s Progressive Matrices and Vocabulary Scales. Oxford, UK: Oxford Psychologists Press SPM1-SPM95.

Reinitz MT, Morrissey J, Demb J (1994) Role of attention in face encoding. J Exp Psychol 20: $161-168$

Reinitz MT, Verfaellie M, Milberg WP (1996) Memory conjunction errors in normal and amnesic subjects. J Mem Lang 35: 286-299

Roberts GMP, Nestor L, Garavan H (2009) Learning and memory deficits in ecstasy users and their neural correlates during a face-learning task. Brain Res 1292: 71-81

Sankoh A J, Huque M F, Dubey SD (1997) Some comments on frequently used multiple endpoint adjustment methods in clinical trials. Stat Med 16: 2529-2542

Schilt T, de Win MML, Jager G, Koeter MW, Ramsey NF, Schmand B, van den Brink W (2008) Specific effects of ecstasy and other illicit drugs on cognition in poly-substance users. Psychol Med 38: 1309-1317. 
Sekine Y, Minabe Y, Ouchi Y, Takei N, Iyo M, Nakamura K, Suzuki, K, Tsukada H, Okada H, Yoshikawa E, Futatsubashi M, Mori N (2003) Association of dopamine transporter loss in the orbitofrontal and dorsolateral prefrontal cortices with methamphetaminerelated psychiatric symptoms. Am J Psychiat 160: 1699-1701

Solowij N, Hall W, Lee N (1992) Recreational MDMA use in Sydney: a profile of 'Ecstasy’ users and their experiences with the drug. Brit J Addict 87: 1161-1172 Tabachnick BG, Fidell LS (2007) Using Multivariate Statistics (Fifth Edition).Boston, Allyn and Bacon

Tanji J, Hoshi E (2001) Behavioral planning in the prefrontal cortex. Curr Opin Neurobiol 11: $164-170$

Thompson PM, Hayashi K, Simon SL, Geauga JA, Hong MS, Sui Y, Lee JY, Toga AW, Ling W, London ED (2004) Structural Abnormalities in the Brains of Human Subjects Who Use Methamphetamine. J Neurosci 24: 6028-6036

Verdejo-García AJ, López-Torrecillas F, Aguilar de Arcos F, Pérez-García M (2005) Differential effects of MDMA, cocaine, and cannabis use severity on distinctive components of the executive functions in polysubstance users: A multiple regression analysis. Addict Behav 30: 89-101.

Wareing M, Fisk JE, Murphy PN, Montgomery C (2004) Verbal working memory deficits in current and previous users of MDMA. Hum Psychopharm Clin 19: 225-234 
Table 1: Age, intelligence, years of education, alcohol and tobacco and illicit drug use by group

\begin{tabular}{|c|c|c|c|c|c|c|c|}
\hline & \multicolumn{3}{|c|}{ Ecstasy Users } & \multicolumn{3}{|c|}{ Nonusers } & \multirow[t]{2}{*}{$\mathrm{p}$} \\
\hline & Mean & $\mathrm{SD}$ & $\mathrm{n}$ & Mean & SD & $\mathrm{n}$ & \\
\hline Age & 22.50 & 2.58 & 44 & 20.96 & 2.25 & 48 & $p=.003$ \\
\hline $\begin{array}{l}\text { Ravens progressive matrices } \\
\text { (maximum 60) }\end{array}$ & 46.98 & 6.43 & 42 & 48.30 & 6.52 & 46 & ns \\
\hline Years of education & 16.71 & 1.95 & 45 & 16.20 & 2.03 & 46 & ns \\
\hline Alcohol (units per week) & 13.63 & 9.13 & 43 & 11.41 & 8.70 & 43 & ns \\
\hline Cigarettes per day & 6.27 & 3.92 & 26 & 3.43 & 3.56 & 7 & na \\
\hline \multicolumn{8}{|l|}{ Total Prior Consumption } \\
\hline Cannabis (joints) & 1451.27 & 2561.16 & 38 & 149.62 & 301.17 & 18 & $\mathrm{p}=.004$ \\
\hline Cocaine (lines) & 804.50 & 1333.21 & 32 & 61.27 & 98.92 & 3 & na \\
\hline Ecstasy (tablets) & 612.98 & 1982.32 & 43 & na & na & 0 & na \\
\hline Ketamine (grams) & 18.74 & 30.14 & 17 & na & na & 0 & na \\
\hline \multicolumn{8}{|c|}{ Long-term Average Dose Per Session } \\
\hline Cannabis (joints) & 2.51 & 1.87 & 38 & 1.36 & 1.01 & 18 & $\mathrm{p}=.004$ \\
\hline Cocaine (lines) & 6.21 & 8.31 & 32 & 2.50 & 1.80 & 3 & na \\
\hline Ecstasy (tablets) & 2.46 & 2.27 & 43 & na & na & 0 & na \\
\hline Ketamine (grams) & .49 & 0.38 & 17 & na & na & 0 & na \\
\hline \multicolumn{8}{|c|}{$\begin{array}{l}\text { Long-term Average Frequency (times } \\
\text { per week) }\end{array}$} \\
\hline Cannabis & 1.15 & 1.32 & 38 & .39 & .62 & 18 & $\mathrm{p}=.005$ \\
\hline Cocaine & .43 & .74 & 32 & .29 & .40 & 3 & na \\
\hline Ecstasy & .44 & .54 & 43 & na & na & 0 & na \\
\hline Ketamine (grams) & .29 & .43 & 17 & na & na & 0 & na \\
\hline \multicolumn{8}{|c|}{$\begin{array}{l}\text { Current Frequency of Use (times per } \\
\text { week) }\end{array}$} \\
\hline Cannabis & 3.15 & 10.12 & 39 & .18 & .32 & 20 & ns \\
\hline Cocaine & .50 & .92 & 33 & .16 & .33 & 5 & na \\
\hline Ecstasy & .22 & .38 & 44 & na & na & 0 & na \\
\hline Ketamine (grams) & .49 & 1.15 & 19 & na & na & 0 & na \\
\hline \multicolumn{8}{|l|}{ Weeks Since Last Use $^{a}$} \\
\hline Cannabis & 8.00 & 33.43 & 41 & 12.00 & 128.00 & 21 & $\mathrm{p}=.076$ \\
\hline Cocaine & 8.00 & 23.00 & 34 & 20.00 & 71.58 & 5 & na \\
\hline Ecstasy & 12.00 & 49.00 & 45 & na & na & 0 & na \\
\hline Ketamine (grams) & 12.00 & 50.50 & 21 & na & na & 0 & na \\
\hline
\end{tabular}

ns: $\mathrm{p}>.05$

na: not applicable

a. Median and interquartile range are reported; p value is for Mann Whitney U 
Table 2: Mean number of old responses to each of the four word pair types (old, conj, item and new) by group

\begin{tabular}{cllll}
\hline & \multicolumn{2}{c}{ Ecstasy Users } & \multicolumn{2}{c}{ Nonusers } \\
\cline { 2 - 5 } & Mean & SD & Mean & SD \\
\hline Single Attention & & & & \\
Hits: Old & 7.70 & 3.11 & 8.43 & 3.28 \\
False Positives & & & & \\
Conjunction & 3.74 & 2.22 & 3.05 & 2.33 \\
Item & 2.30 & 1.55 & 2.43 & 2.40 \\
New & 1.70 & 1.84 & 1.43 & 1.63 \\
Signal Detection & & & & \\
D Prime $^{\mathrm{a}}$ & 1.10 & .83 & 1.38 & 1.05 \\
Criterion $^{\mathrm{b}}$ & .48 & .34 & .51 & .33 \\
& & & & \\
Divided Attention & & & & \\
Hits: Old & 7.73 & 2.71 & 6.52 & 2.53 \\
False Positives & & & & \\
Conjunction & 5.50 & 2.35 & 3.74 & 1.75 \\
Item & 4.50 & 2.82 & 3.74 & 2.43 \\
New & 4.32 & 2.40 & 3.19 & 1.86 \\
Signal Detection & & & & \\
D Prime $^{\mathrm{a}}$ & .58 & .66 & .58 & .55 \\
Criterion $^{\mathrm{b}}$ & .24 & .37 & .48 & .32 \\
\hline
\end{tabular}

a: $z(H)-z(F)$ where $H$ is defined as the proportion of correct responses and $F$ the proportion of false positive responses.

b: $-[z(H)+z(F)] / 2$ 
Table 3: Correlations between measures and indices of long-term drug use

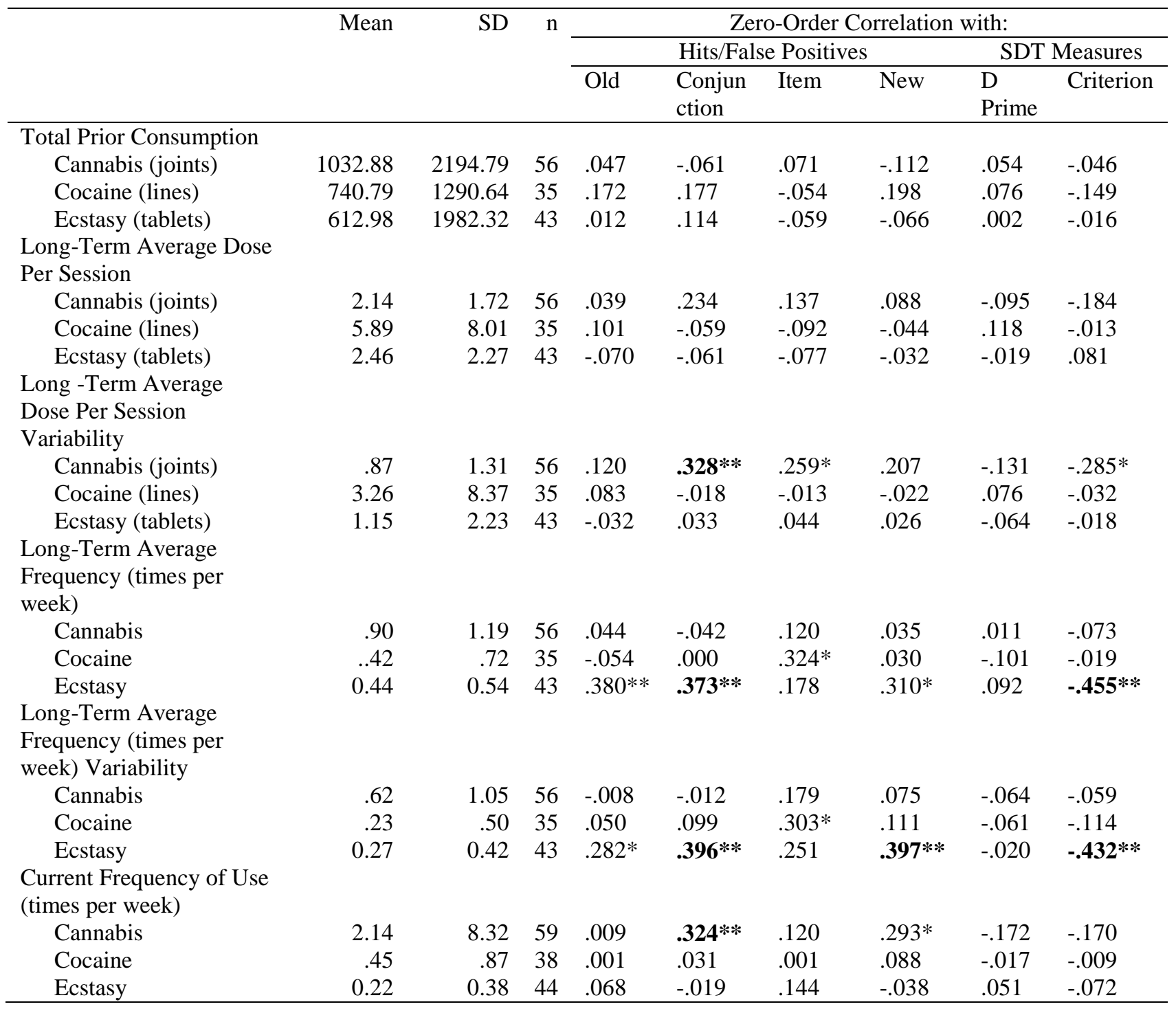

$* * * \mathrm{p}<.001 ; * * \mathrm{p}<.01 ; * \mathrm{p}<.05$, one tailed 
Table 4. Partial correlation controlling for the effects of other drugs with significant zero order correlations.

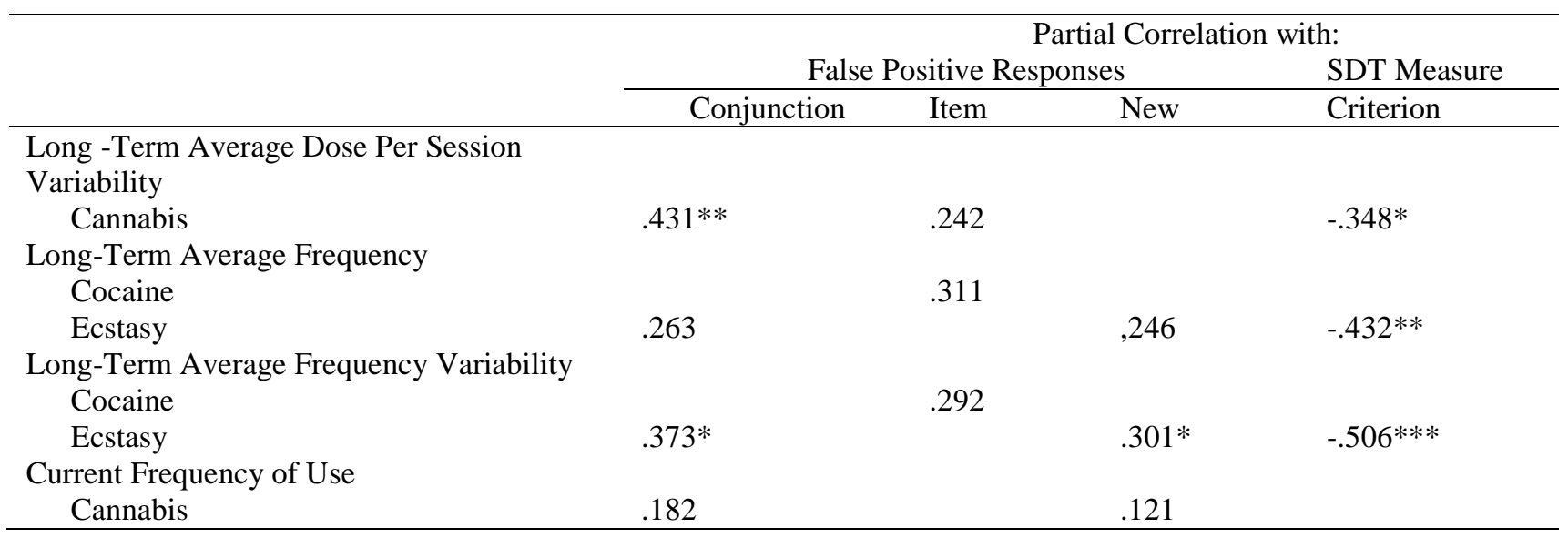

${ }^{* * *} \mathrm{p}<.001 ; * * \mathrm{p}<.01 ; * \mathrm{p}<.05$, one tailed 
Figure 1: False Positive Responses by Task Condition and Drug Use

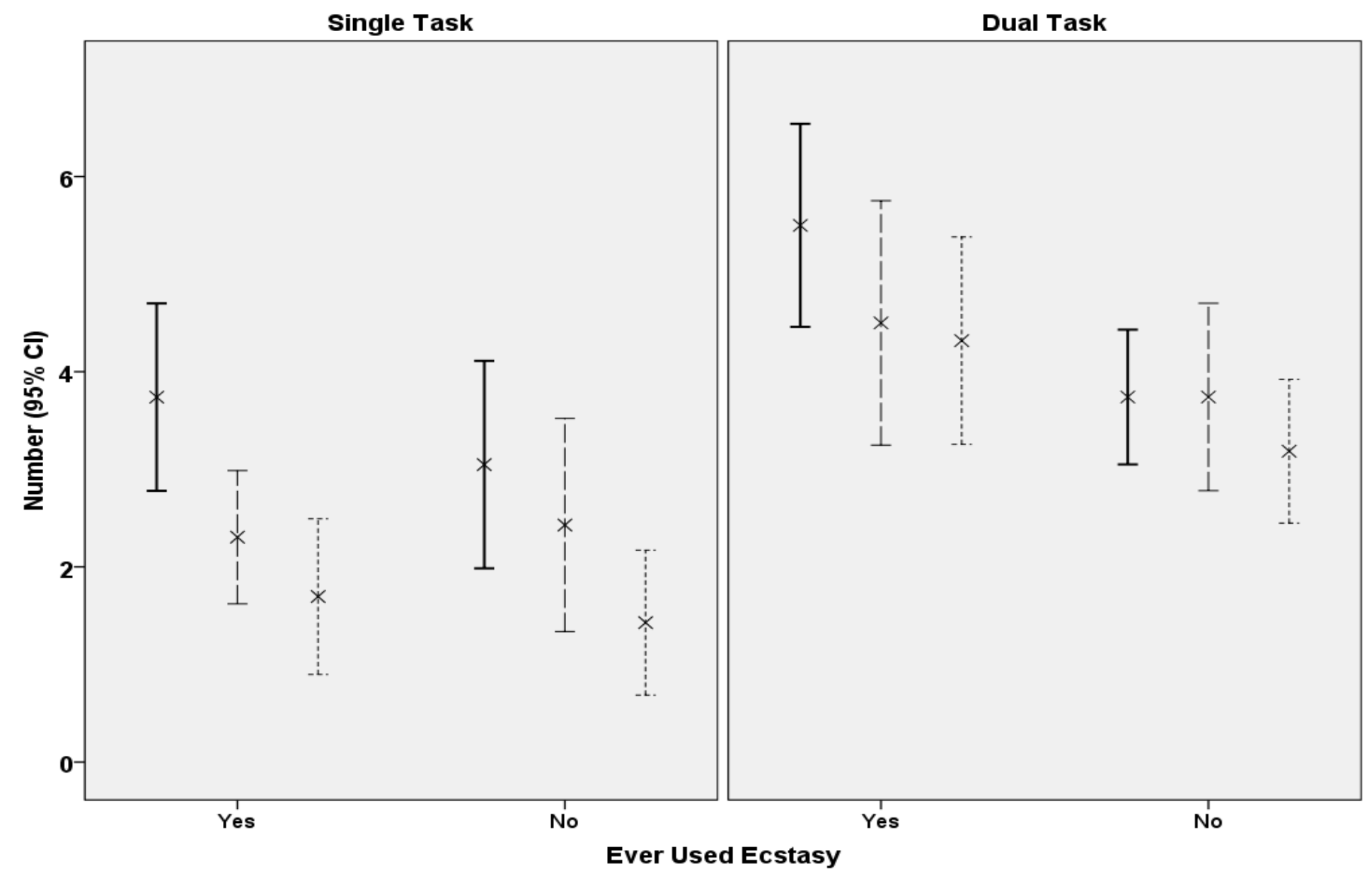

I Conjuntion Word Pairs

I Item Word Pairs

New Word Pairs 